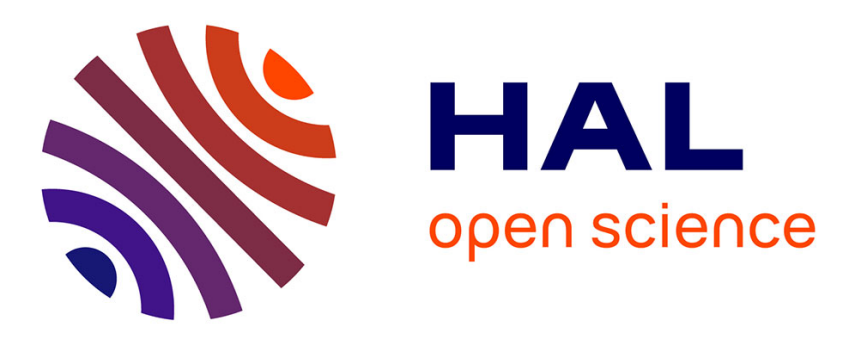

\title{
Ultrafast switching of surface plasmonic conditions in nonplasmonic metals
}

Emile Bévillon, Jean-Philippe Colombier, Vanina Recoules, Hao Zhang, Chen Li, Razvan Stoian

\section{- To cite this version:}

Emile Bévillon, Jean-Philippe Colombier, Vanina Recoules, Hao Zhang, Chen Li, et al.. Ultrafast switching of surface plasmonic conditions in nonplasmonic metals. Physical Review B: Condensed Matter and Materials Physics (1998-2015), 2016, 93, pp.165416. 10.1103/PhysRevB.93.165416 . ujm01340983

\section{HAL Id: ujm-01340983}

\section{https://hal-ujm.archives-ouvertes.fr/ujm-01340983}

Submitted on 3 Jul 2016

HAL is a multi-disciplinary open access archive for the deposit and dissemination of scientific research documents, whether they are published or not. The documents may come from teaching and research institutions in France or abroad, or from public or private research centers.
L'archive ouverte pluridisciplinaire HAL, est destinée au dépôt et à la diffusion de documents scientifiques de niveau recherche, publiés ou non, émanant des établissements d'enseignement et de recherche français ou étrangers, des laboratoires publics ou privés. 


\title{
Ultrafast switching of surface plasmonic conditions in nonplasmonic metals
}

\author{
E. Bévillon, ${ }^{1}$ J. P. Colombier, ${ }^{1,}{ }^{*}$ V. Recoules, ${ }^{2}$ H. Zhang, ${ }^{1}$ C. Li, ${ }^{1}$ and R. Stoian ${ }^{1}$ \\ ${ }^{1}$ Laboratoire Hubert Curien, UMR CNRS 5516, Université de Lyon, Université Jean-Monnet, 42000 Saint-Etienne, France \\ ${ }^{2}$ CEA-DIF, 91297 Arpajon, France
}

(Received 11 September 2015; revised manuscript received 22 March 2016; published 14 April 2016)

\begin{abstract}
We demonstrate that ultrafast carrier excitation can drastically affect electronic structures in nonplasmonic metals and determine a transient, brief surface plasmonic state, potentially creating the conditions for a plasmonic switch. The initial state can be related to $d$-band partial filling and splitting, with a pseudo-band-gap accommodating the chemical potential. This determines a quasi-resonant-like spectral behavior of the optical constants for pumping carriers across the $d$-band pseudogap, i.e., visible frequencies. The relation between real and imaginary parts of the refractive index does not fulfill surface plasmonic conditions in the visible photon range. Using first-principles molecular dynamics and Kubo-Greenwood formalism for laser-excited tungsten we show that carrier heating mobilizes $d$ electrons into collective inter- and intraband transitions leading to a sign flip in the imaginary optical conductivity, activating plasmonic properties for the initial nonplasmonic phase. The drive for the laser-induced optical evolution in this case does not rely on a variation of the free electron number but can be visualized as an increasingly damped character of the quasiresonance at visible frequencies. Here laser heating determines an energy-dependent degree of occupation with broadening profiles. The subsequent evolution of optical indices for the excited material is confirmed by time-resolved ultrafast ellipsometry. The large optical tunability extends the existence spectral domain of surface plasmons in ranges typically claimed in laser self-organized nanostructuring. Nonequilibrium heating is thus a strong factor for engineering optical control of evanescent excitation waves, particularly important in laser nanostructuring strategies.
\end{abstract}

DOI: 10.1103/PhysRevB.93.165416

\section{INTRODUCTION}

Collective nonequilibrium effects on transport properties in metallic systems are topics of current interest as nonstandard behaviors play an increasing role in optics, thermodynamics, or magnetism. Ultrafast laser electronic heating can mobilize localized states, inducing massive nonlinear contributions to optical and thermal transport or structural metastability. Understanding the intimate electronic mechanisms of optical coupling in excited solids and controlling excitation transients is therefore of prime importance as new applications are emerging in ultrafast optics, plasmonics, and heat control strategies. The rapid laser excitation effects are primarily related to the evolution of free carriers and they can be seen in optical and transport properties. If consequences for transport coefficients are readily expectable [1,2], and the role of nonequilibrium electronic transfer was early recognized [3,4], subtle atomistic effects can be inferred at the level of the band structure with unexpectedly strong consequences. Charge transfer and carrier screening during nonequilibrium concur to a dynamic adjustment of thermal and optical properties to accommodate swift excitation. In this respect, unconventional structural and electronic dynamics were already observed. Recoules et al. [5] showed that the participation of localized $d$ orbitals in the excitation process in noble metals enforces the ion-ion potential and ensures a stronger lattice cohesion. This determines a hardening of the phonon modes and an increased resistance against nonequilibrium melting. Oppositely, for a range of transition metals, nonequilibrium electronic excitation can facilitate the conditions for melting, lowering the thermodynamic point

\footnotetext{
*jean.philippe.colombier@univ-st-etienne.fr
}

and thus accelerating the phase transition [6]. In general, nonequilibrium charge supply from $d$-band reservoirs allows large variations of thermal characteristics in transition metals [7], improving thus energy and thermal transport [8]. Moreover, nonthermal carrier distributions before Fermi-like equilibration can restrict the collisional phase space around the Fermi level, severely damping electron-phonon coupling [9] and therefore the process of energy transfer to the atomic lattice. Smeared carrier distributions at increasing electronic temperatures are equally effective in the Pauli unblocking of available states for interband transitions, triggering thus strong nonlinearities in metallic systems [10,11]. Furthermore, electronic occupation of delocalized states and filling from localized reservoirs (e.g., $d$ bands in transition metals) redefine classical views on the definition of free electron density [12], introducing significant dynamics in optical behaviors. Tuning ultrafast optical response via electronic reactions was thus proposed for active large bandwidth modulation in ultrafast plasmonics [13]. Engineering macroscopic optical response on ultrafast scales equally relates to the onset of optical resonances [14,15] on excited surfaces with spectral and spatial disturbances, e.g., Wood's anomalies. One debated example concerns ultrafast laser nanostructuring of solids in self-assembled regular patterns. The universal phenomenonobserved half a century ago [16] — carries application potential in functional surfaces [17], color-coded optical traceability and multidimensional information storage $[18,19]$, or feedbackdriven nanolithography [20]. Key questions in laser-induced periodic surface structures (LIPSS) concern the origin of spatially modulated energy patterns [21] and the interplay between optical resonances and nonlinear feedback. In the frame of a scattering model, electromagnetic calculations indicate the involvement of surface waves inducing collective motion [21]. Among them, the surface plasmon 

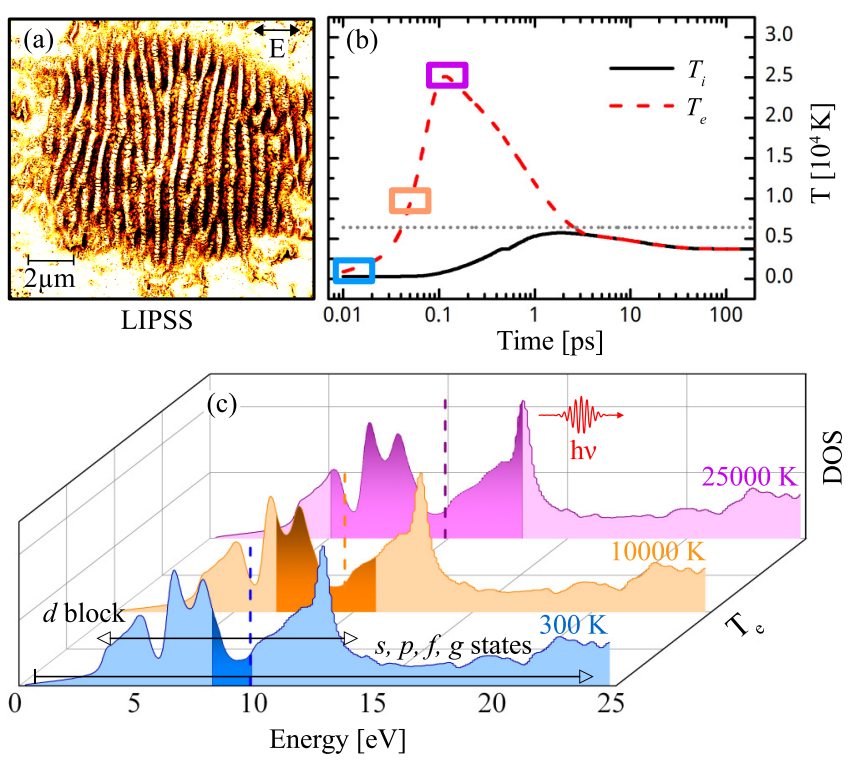

FIG. 1. (a) Typical field-perpendicular surface periodic nanostructures at quasi-wavelength periodicity induced by $800 \mathrm{~nm}, 50 \mathrm{fs}$ laser pulses on W. (b) Electron (dashed red) and lattice (solid black) temperature evolutions for $\mathrm{W}$ irradiated by a $50 \mathrm{fs}$ laser pulse in the vicinity of the ablation threshold. Dotted line indicates the standard vaporization limit. (c) DOS of bcc W computed at $T_{i}=300 \mathrm{~K}$ and its evolution with $T_{e}$. Dashed lines indicate the electronic chemical potential, and dark colored areas show occupied electronic states mainly impacted by $800 \mathrm{~nm}$ photons.

(SP) with its ability to enhance electromagnetic energy on nanoscales holds a specific and largely discussed role. It has been recently argued [22] that a potential plasmonic wave, though not necessarily at the origin of the phenomenon, may enhance the contrast of a modulated energy deposition pattern within a general scattering approach [21], contributing thus to the ability of generating high contrast and regular corrugation.

The potential involvement of SP interference in laser nanostructuring is in close relation to surface optical indices. Thus the experimentally observed onset of polarization-dependent periodic structures for nonplasmonic metals, as demonstrated on tungsten [23], is intriguing (in the plasmonic hypothesis) in optical ranges theoretically forbidden by predictions based on ambient optical indices. A typical example of ultrafast LIPSS at $800 \mathrm{~nm}$ is provided in Fig. 1(a), showing quasiwavelength periodicity with a well contrasted definition. The positive real permittivity of $\mathrm{W}$ precludes normally the generation of electronic surface waves and plasmon polariton coupling. The possibility to excite collective material motion pinpoints to a more intricate dynamic response of the system. W shows a non-Drude-like character with both free and localized electronic contributions to the dielectric function, giving a nonplasmonic state at room temperature. We investigate the impact of ultrafast heating on electronic structures capable of initiating a large excursion of optical properties, namely an excitation-driven plasmonic state in otherwise nonplasmonic metal. First-principles approaches are chosen to interrogate electronic-driven evolution of optical properties in tungsten and their consequences on plasmonic behaviors at time and spatial scales difficult to access by experiments.

The paper is structured as follows. We first present the calculation method that interrogates the linear response of the dielectric function which, via the electronic temperature, evolves nonlinearly with the pump energy. We characterize the electronic structure in the initial state and discuss the effect of electronic heating and redistribution within the various electronic states. We demonstrate the associated evolution of optical indices towards fulfilling surface plasmonic conditions for a brief moment, comparable to the pulse duration, and argue that the electronic redistribution around the chemical potential located in a $d$-band pseudogap is paramount in instrumenting the transition with specific inter- and intraband evolutions. The behavior is then experimentally verified using time-resolved ellipsometry.

\section{CAlCulation Details}

The calculations correspond to ultrashort pulse irradiation conditions enabling LIPSS around the damage threshold ( $90 \mathrm{~mJ} \mathrm{~cm}^{-2}$ absorbed fluence). Transient electron and lattice temperatures in $\mathrm{W}$ for single ultrashort pulse irradiation [Fig. 1(b)] are estimated using a two-temperature hydrodynamic code (Esther) [24] which describes the energy balance using Helmholtz optical formalism and electron-phonon relaxation with temperature-dependent transport properties $[7,12]$. Electronic temperatures on the rising heat cycle can amount to $2.5 \times 10^{4} \mathrm{~K}$, inducing electron-phonon nonequilibrium for up to $3 \mathrm{ps}$, with the material in solid state for at least $1 \mathrm{ps}$. Internal electronic thermalization is assumed, justified by the high levels of energy deposition in the vicinity of the threshold which accelerate electronic energy exchange to fs scales [9]. In this range, electronic calculations are carried out with the plane-wave code ABINIT [25], in the frame of the density functional theory [26,27] extended to finite electronic temperatures [28]. The generalized gradient approximation [29] is employed to model exchange and correlation energies, and projector augmented wave atomic data [30] account for the effects of nuclei and core electrons. A 54-atom supercell of body-centered-cubic (bcc) W is considered, with a $5 s^{2} 5 p^{6} 5 d^{4} 6 s^{2}$ valence electronic configuration; $4 f$ electrons are neglected as their effect is weak at the $T_{e}$ considered here [12]. We first perform $a b$ initio molecular dynamics simulations in the isokinetic ensemble at room temperature, with $T_{i}=T_{e}=300 \mathrm{~K}$ during $2 \mathrm{ps}$. This calculation provides an average thermodynamic equilibrium from which ionic configurations are extracted, standing for representative states of the $\mathrm{W}$ lattice at ambient conditions. Then electronic structures are computed at $T_{e}$ of $300,10^{4}$, and $2.5 \times 10^{4} \mathrm{~K}$. They serve as a basis to evaluate optical properties in the solid phase at these levels of electronic excitation.

\section{RESULTS}

\section{A. Evolution of the electronic structure}

The primary laser excitation is related to an elevation of the electronic temperature. We therefore first focus on the evolution of the electronic density of states (DOS) with 
electronic temperatures in the presence of a cold, ambient lattice, with calculated DOS profiles provided in Fig. 1(c). This is justified for the initial time domain, comparable to the pulse duration, where excitation determines electronic heating, before significant transfer towards the lattice takes place. If finite ionic temperature induces a certain smoothing of the features compared to calculations at $T_{i}=0 \mathrm{~K}$ [12] due to a loss of degeneracy arising from atom oscillations around their high-symmetry position, a highly structured $d$-band profile remains. The DOS consists of a split partially filled $d$ block of high density of states located between 0 and $15 \mathrm{eV}$, combined with a rough square root distribution profile of $s p f g$ states with quasi-free-electron behavior. For the nonexcited material, the location of the chemical potential within a midrange pseudogap between high density of filled and empty electronic states determines the nonplasmonic behavior in the $1-5 \mathrm{eV}$ range, i.e., for visible photon energies bridging the gap, emulating to a certain extent a "gaplike" character. This determines, as will be seen later in the text, a specific quasiresonant character of the optical response. The effect of laser heating is then potentially observable in an evolution of the electronic profile or its degree of occupation. The DOS shape is remarkably stable against electronic heating with a maximal $d$-block shift of $0.15 \mathrm{eV}$ at $2.5 \times 10^{4} \mathrm{~K}$, indicative of a stiff electronic structure. This relates to the roughly symmetric profile of the DOS on both sides of the electronic chemical potential $\mu$. The high density of empty electronic states on the right side of $\mu$ collects the excited electrons from the left-sided filled electronic states, leading to a weak $T_{e}$ dependence of $\mu$, contrary to most transition metals $[7,12]$. As the DOS does not change, the increase of $T_{e}$ mainly determines, via an evolution of the distribution function, electronic transfer from filled to empty $d$-electronic states; however an additional small transfer from $s p$ to $d$ electronic states also occurs, leading to a slight increase of the total number of $d$ electrons with $T_{e}$. These effects induce a moderate strengthening of the localization degree of the charge density and, subsequently, this determines a weak augmentation of the electronic screening as confirmed by the Hartree energy increase [12]. This implies a certain stability of the structural order and a dynamic evolution of optical properties. The main $T_{e}$ effect resumes then to a Fermi broadening within the symmetric DOS distribution around the chemical potential, and the impact of the occupation probability is discussed below.

\section{B. Evolution of the optical properties}

Relying on the as-determined electronic structures, the subsequent optical properties are obtained from an average on three ionic configurations. The real part of the frequencydependent optical conductivity is obtained within the KuboGreenwood (KG) formalism [31]:

$$
\begin{aligned}
\sigma_{r}(\omega)= & \frac{2 \pi}{3 \omega \Omega} \sum_{i, j, k} \sum_{\alpha=1}^{3}\left[f\left(\epsilon_{i, k}\right)-f\left(\epsilon_{j, k}\right)\right] \\
& \times\left|\left\langle\Psi_{j, k}\left|\nabla_{\alpha}\right| \Psi_{i, k}\right\rangle\right|^{2} \delta\left(\epsilon_{j, k}-\epsilon_{i, k}-\hbar \omega\right),
\end{aligned}
$$

where electronic transitions from $i$ to $j$ states are integrated over the reciprocal space for each photon energy $\hbar \omega$, account-

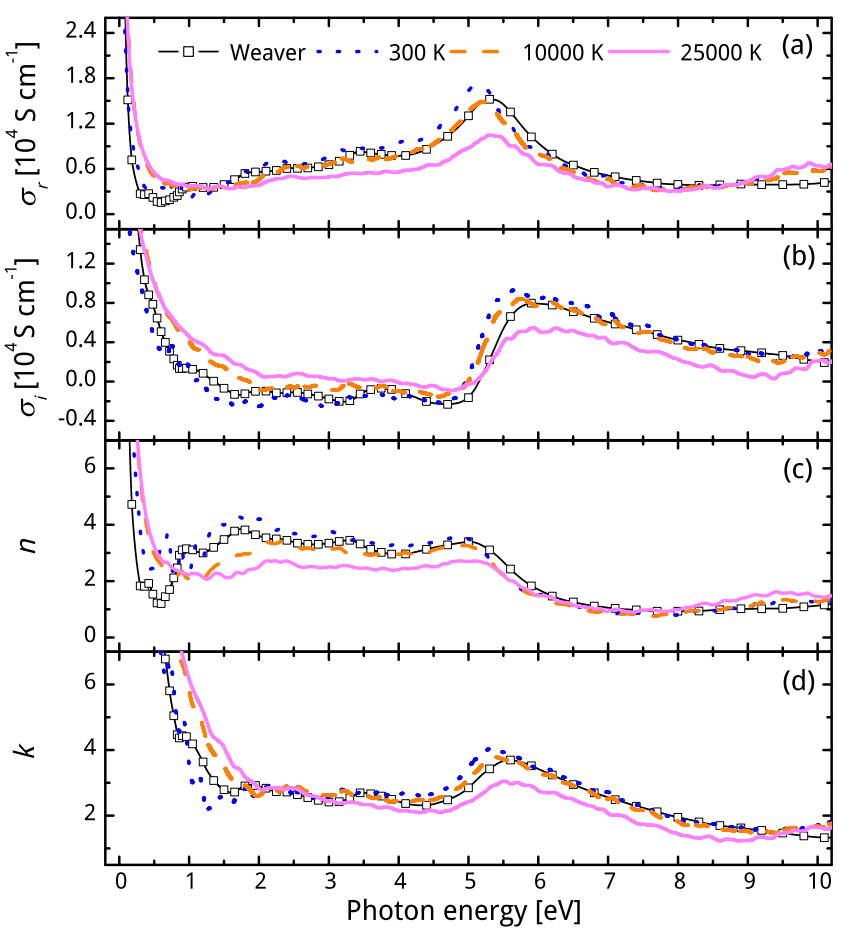

FIG. 2. Real (a) and imaginary (b) part of the frequencydependent optical conductivity; real (c) and imaginary (d) part of the optical indices. Colored curves stand for the electronic temperatures of $300 \mathrm{~K}, 10^{4} \mathrm{~K}$, and $2.5 \times 10^{4} \mathrm{~K}$ and the squares represent the experimental data for nonexcited $\mathrm{W}$ [33].

ing for the Fermi-Dirac occupations $f(\epsilon)$ and the eigenenergies of the electronic states $\epsilon . \nabla$ represents the velocity operator. $\Omega$ is the volume of the cell while $\alpha$ corresponds to the three spatial dimensions. The imaginary part of the frequency-dependent conductivity is obtained using the Kramers-Kronig (KK) relation, $\sigma_{i}(\omega)=-\frac{2}{\pi} \mathcal{P} \int_{0}^{\infty} \frac{\sigma_{r}\left(\omega^{\prime}\right) \omega}{\omega^{\prime 2}-\omega^{2}} d \omega^{\prime}$, where $\mathcal{P}$ is the principal value of the integral. Frequency-dependent permittivities and optical indices can be derived.

Optical properties follow the evolutions in the electronic properties and show a dynamics that is affected by the electronic temperature and therefore by the pumping power [32]. Rather large optical excursions are argued below as originating in an electronic redistribution effect and, for a certain value of electronic heating, shown to lead to the establishment of plasmonic states. Figure 2 shows the computed frequencydependent optical conductivities $\tilde{\sigma}=\sigma_{r}+i \sigma_{i}$ and optical indices $\tilde{n}=n+i k$ as a function of the electronic temperature. A good agreement is found between theoretical values obtained at $300 \mathrm{~K}$ and the reflectivity measurements of Weaver [33], confirming a realistic description of the $\mathrm{W}$ electronic structure. $\sigma_{r}$ is an implicit measure of the optical absorption and its spectral behavior can be inferred based on the profile of the energy bands calculated in Fig. 1(c). For the low $-T_{e}$ case (dotted blue line), at photon energies below $0.5 \mathrm{eV}$, the intraband part dominates, resulting from electronic transitions inside $6 s p$ bands. This contribution rapidly decays as the photon energy increases. On the other hand, interband transitions originating from the partially filled $5 d$ subbands become gradually more important [34]. From 0.5 to $5.1 \mathrm{eV}$, 
photon-driven electronic transitions access an increasingly larger DOS domain $( \pm h v)$ centered on $\mu$, resulting in a stepwise build-up of $\sigma_{r}(\omega)$, mapping the local DOS. This trend can be seen as the wing slope of a resonant behavior around the $5.1 \mathrm{eV}$ peak given by the particular electronic DOS splitting around the Fermi level and the finite width of the sidebands, as suggested earlier in the text. The conductivity finally decreases once the main $d$-band peaks have been included and diluted into the continuously increasing electronic transition phase space. From the KK relation, $\sigma_{i}(\omega)$ relies on the overall $\sigma_{r}(\omega)$ profile. Conceptually, this integral can be separated into two parts depending on the value of $\omega^{\prime}$ with respect to the reference value $\omega$. For $\omega^{\prime} \in[0, \omega[$, the integral is negative and reverse signs for higher $\omega^{\prime}$. Accordingly, the magnitude of $\sigma_{i}$ depends on the profile of the real conductivity mainly around $\omega$. A quasisymmetric profile of $\sigma_{r}$ tends to balance positive and negative components of the integral and provide low values for $\sigma_{i}$. On the contrary, an asymmetric profile emphasizes the respective sign components of the integral, depending on the trend of asymmetry (left or right turned), determining positive or negative values of the imaginary conductivity. For W, the positive slope of the $\sigma_{r}$ profile from 1 to $5.1 \mathrm{eV}$ with values ranging from $0.5 \times 10^{4}$ to $1.7 \times 10^{4} \mathrm{~S} \mathrm{~cm}^{-1}$ leads to a negative $\sigma_{i}(\omega)$ at ambient conditions. A spectral view on $\sigma_{i}(\omega)$ indicates an anomalous-like dispersive behavior related to the absorption resonances.

The profiles of optical conductivities in Figs. 2(a) and 2(b) and optical indices in Figs. 2(c) and 2(d) significantly change with the electronic temperature, requiring an introspection in the corresponding excitation-driven electronic effects. If the observed changes in the optical properties are not a consequence of DOS variation due to its stability against heating, the electronic temperature affects the degree of filling around the chemical potential. Thus the optical evolution is the direct consequence of a broadened Fermi-Dirac charge redistribution within and around the pseudogap, making more states available for photon-induced transitions. This equally damps the resonant-like character from the $d$-band splitting on the optical response. With the elevation of $T_{e}$, the optical transitions concern an increasing $\mu$-centered interval mainly corresponding to [ $-h v-\frac{3}{2} k T_{e}, h v+\frac{3}{2} k T_{e}$ ] [35], illustrated in Fig. 1(c) for a photon energy of $1.55 \mathrm{eV}$. The consequence is manifold, observable in the $\sigma_{r}$ which maps the spectral absorption. First this produces a broadening of available transition phase space for the various photon energies. This translates into an increase of the intraband part, balanced by a decrease in the interband domain (qualitatively similar to a clockwise turn), damping the $\sigma_{r} 5.1 \mathrm{eV}$ peak. The subsequent asymmetric change of slope in $\sigma_{r}$ with $T_{e}$ determines the increase of $\sigma_{i}$ in the $1-5 \mathrm{eV}$ (as the dispersive behavior across the resonance flattens) and a passage in the positive values domain for a significant part of the spectral domain. The situation becomes more clear in the evolution of the optical indices. At $1.55 \mathrm{eV}(800 \mathrm{~nm})$, relevant for the LIPSS, optical indices are strongly affected by the heating of the electronic system, with $\tilde{n}=4.0+2.4 i$ at $3 \times 10^{2} \mathrm{~K}$ reaching $\tilde{n}=2.1+3.8 i$ at $2.5 \times 10^{4} \mathrm{~K}$. Thus, in the nearinfrared and low-frequency visible part of the spectrum, the real part of the index goes down and the imaginary part augments.

\section{EXPERIMENTAL OBSERVATION OF THE OPTICAL DYNAMICS}

The predicted evolution was confirmed by time-resolved pump-probe ultrafast ellipsometry on laser-excited W surfaces. The transient optical properties upon ultrafast (120 fs) laser irradiation were probed at $1.55 \mathrm{eV}$ photon energy using a two-angle one-color time-resolved ellipsometry method following the technique proposed in Ref. [36]. The static properties of the nonexcited surface were first evaluated $e x$ situ using a commercial ellipsometer (Uvisel, Horiba Jobin Yvon) and the results give $\tilde{n}=3.57+i 3.15$ for massive $\mathrm{W}$ materials (Goodfellow 99.95\% purity, mechanically polished). Alongside massive $\mathrm{W}$, foils (Goodfellow $0.3 \mathrm{~mm}$ thick, polished at $0.1 \mathrm{mRa}$, purity $99.95 \%$ ) were equally used as reference. The dynamic reflectivity changes were subsequently interrogated on massive thick targets by p-polarized $120 \mathrm{fs}$, $800 \mathrm{~nm}$ low-energy nonperturbing probe laser pulses at $27.1^{\circ}$ and $65.8^{\circ}$ incidence angles. The probe pulses were time synchronized with fs accuracy with the exciting pump pulse of equally $120 \mathrm{fs} 800 \mathrm{~nm}$, arriving at normal incidence on the $\mathrm{W}$ surface. Two photodiode detectors were used in imaging geometries with respect to the surface. The probed zone is significantly smaller than the spatial extent of the excited region. The exciting fluences were chosen slightly below the ablation threshold.

The swift optical activity on massive $\mathrm{W}$ samples is underlined by strong reflectivity changes, with a reflectivity snapshot example for an input peak fluence of $0.12 \mathrm{~J} / \mathrm{cm}^{2}$, just below damage threshold being shown in Fig. 3(a). The time profile of the reflectivity transients maps the heating/cooling cycle of the electronic system and the associated redistribution in the electronic occupation around the chemical potential with $T_{e}$. The measured maximum values of the transient reflectivity changes occurring just after the peak of the excitation pulse were then depicted in terms of corresponding $(n, k)$ values in the $(n, k)$ optical phase space. They represent uniquely determined $(n, k)$ pairs obtained by inverting Fresnel formulas at the given angles. The result is presented in Fig. 3(b) and the dynamic range of these values almost coincides with the pulse duration. The intersection point of the $(n, k)$ contour plots
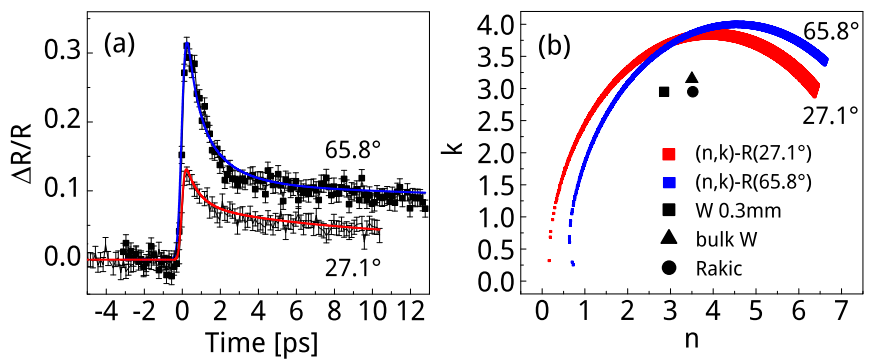

FIG. 3. (a) Example of dynamic time-resolved reflectivity traces at the two probe angles at incident pump peak fluence of $0.12 \mathrm{~J} / \mathrm{cm}^{2}$. (b) $(n, k)$ contour plots corresponding to measured reflectivities at the two given angles. The intersection point represents a uniquely determined $(n, k)$ pair satisfying simultaneously the two reflectivity conditions. Nonexcited values obtained from ellipsometric measurements on bulk and foil samples as well as literature data [37] are given as references. 
corresponding to two angle-resolved measured reflectivities were used to extract the corresponding $(n, k)$ pair, allowing thus access to the evolution of optical indices. The result is $\tilde{n}=$ $3.21+i 3.77$, where resulting optical indices indicate slight $n$ decrease and increase in $k$ above the experimental value of its dispersive part. Note that these indices are calculated at the peak of excitation and correspond to a macroscopic state averaged over a region set by the optical penetration depth of $18 \mathrm{~nm}$, with inhomogeneous temperature distribution. The accuracy of the measurement is affected by the roughness and local planarity of the surface and care was taken to minimize the errors. The optical evolution has strong impact on the possibility to excite surface plasmons, with the fulfillment of the required optical conditions.

For air-material interfaces the condition for surface plasmon existence reduces to $\varepsilon_{r}=n^{2}-k^{2}<-1$. This requirement is therefore fulfilled at fluences in the close vicinity of the damage threshold reflecting the experimental situation in Fig. 3 and the calculation results in Figs. 2(c) and 2(d), marking thus the ultrafast plasmonic activation. This activation is as fast as the laser pulse and quasisynchronized with it. After this, the electronic relaxation and lattice heating will switch off the effect.

\section{DISCUSSION}

\section{A. The achievement of a plasmonic state}

We propose below a discussion on the consequences of the activation of the condition for plasmon existence (note that the actual plasmon excitation would require in addition the fulfilling of synchronism conditions). Surface plasmon periodicity $\lambda_{\mathrm{sp}}$ as a function of laser wavelength $\lambda$ is given by $\lambda_{\text {sp }}=\lambda / \eta$, where $\eta=\operatorname{Re}\left\{\left[\tilde{n}^{2} /\left(\tilde{n}^{2}+1\right)\right]^{1 / 2}\right\}$ is the real part of the effective refractive index [38]. Calculated $\eta$ is plotted in Fig. 4 and the absence of data indicates nonexistence domains of surface plasmons. With the increase of $T_{e}$, one can note the expansion of the existence domain in the red photon energy region. The temperature-induced broadening of the electronic transition domain determines thus the conditions for a plasmonic switch at visible optical frequencies. It appears that, upon electronic excitation, the light-induced onset of

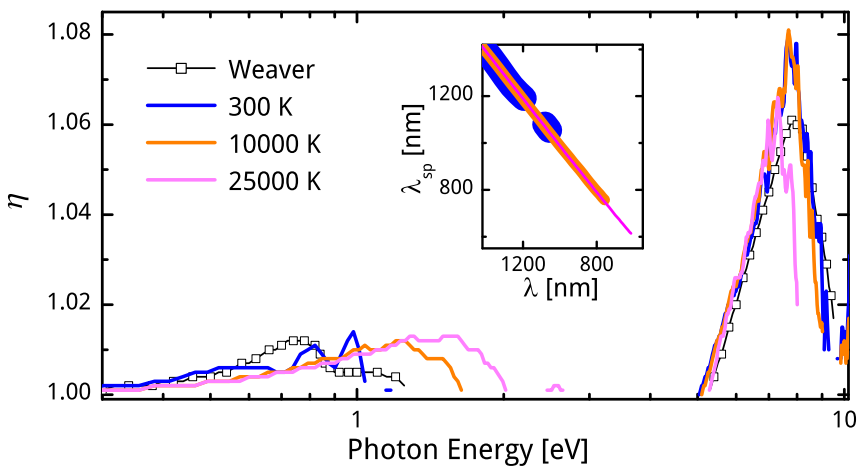

FIG. 4. Real part of the effective refractive indices $(\eta)$ in case of air-tungsten interface as a function of $T_{e}$. The symbol line stands for experimental data. Inset: Variation of plasmon wavelength as a function of laser wavelength in a spectral domain ranging from 0.9 to $2.3 \mathrm{eV}$.

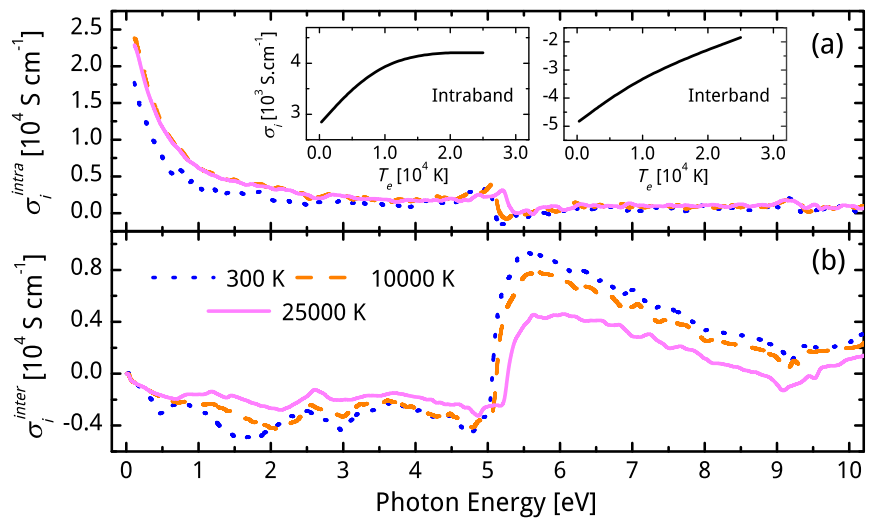

FIG. 5. Intra- and interband contribution to the imaginary conductivity as a function of $T_{e}$. Inset: Local evolution of $\sigma_{i}\left(T_{e}\right)$ at $1.55 \mathrm{eV}$

plasmonic character can sustain a contribution based on optical resonances for LIPSS at 800nm even though room temperature optical indices indicate nonplasmonic properties. Quantifying this contribution is not the object of this paper, but, arguably, within a set of interacting optical and electronic surface waves [22], the contrast of LIPSS is reinforced. As expected within a general scattering frame, for air-metal interfaces $\eta$ remains close to 1 , especially at low photon energy, leading to $\lambda_{\mathrm{sp}}$ slightly inferior to the laser wavelength (inset of Fig. 4).

\section{B. Interband and intraband contributions}

From the expression of the dielectric permittivity $\tilde{\varepsilon}=1+$ $i \tilde{\sigma} / \omega \varepsilon_{0}$ the existence condition can be expressed more directly in terms of imaginary conductivity (and implicitly on real permittivity) with $\sigma_{i}>2 \omega \varepsilon_{0}$. To clarify processes leading to the increase of the existence domain, we propose a simplifying approach. The imaginary conductivity is tentatively split into intra- and interband components as depicted in Figs. 5(a) and 5(b). We first compute optical properties for a degenerate electronic system at $T_{i}=0 \mathrm{~K}$ and $T_{e}=300,10^{4}$, and $2.5 \times 10^{4} \mathrm{~K}$. This disregards ionic temperature and phonon effects, assuming they assist mostly the intraband component via momentum conservation conditions. Conceptually this is justified in a classical view by the temperature dependence of damping frequencies. Accordingly, the intraband contribution vanishes and $\sigma_{i}^{\text {inter }} \simeq \sigma_{i}\left(T_{i}=0 \mathrm{~K}\right)[$ Fig. 5(b)]. The intraband part at $T_{i}=300 \mathrm{~K}$ is then extracted by subtracting the asdetermined $T_{i}$-insensitive interband part from the total value of the imaginary conductivity [Fig. 2(b)]. In the visible spectral range, the intraband part $\sigma_{i}^{i n t r a}$ rapidly increases with the rise of $T_{e}$ and saturates at $10^{4} \mathrm{~K}$. An example of this behavior at $1.55 \mathrm{eV}$ is given in the inset. At the opposite, the interband part shows a more complex behavior, with negative values of $\sigma_{i}^{\text {inter }}$ in the 0 to $5 \mathrm{eV}$ energy interval that leads to negative values of the total imaginary conductivity, explaining the nonplasmonic nature of this metal. At higher energies, a resonance-like dispersive shape induced by the $5.1 \mathrm{eV}$ peak of $\sigma_{r}$ is clearly visible [Fig. 5(b)]. By weakening the peak of $\sigma_{r}$, Fermi broadening also flattens the shape of $\sigma_{i}^{\text {inter }}$ around the resonance and gradually reduces its negative component, progressively switching on plasmonic properties 
of W. The increase is not yet saturated at $2.5 \times 10^{4} \mathrm{~K}$ giving the possibility of a stronger lift and a subsequent extension of the plasmon existence domain for higher electronic temperatures. This highlights the preponderant role of $d$-electron-driven interband transitions in the optical response at high $T_{e}$.

We note that, even if it involves a classical concept of electronic redistribution via Fermi smearing, the optical evolution is nontrivially related to the presence of partially filled $d$ bands accommodating the chemical potential in a pseudogap. It does not relate to evolutions in population number or collision frequency. The collision frequency related to the intraband part could be expressed within the approximation of a Drude model as $v=\omega \sigma_{r}^{\text {intra }} / \sigma_{i}^{\text {intra }}$ [39]. The calculated evolution as a function of the electronic temperature in our domain of interest shows a less than moderate increase of up to $25 \%$ from $1.95 \times$ $10^{14}$ to $2.48 \times 10^{14} \mathrm{~Hz}$. Thus the typical evolution factors in semiclassical approaches are not driving elements in this case. This quasiconstant evolution of collision frequencies, in combination with a weakly varying free electron number [12], stresses that the Fermi smearing redistribution of spatially localized density of charges within a structured partially filled $d$ block is the main mechanism responsible for these transient optical evolutions and plasmonic switch conditions. Thus, for tungsten and related materials, considering the characteristics of the band structure becomes critical, beyond standard model approaches [37].

The electronic evolution described above is related to the fast achievement of the nonequilibrium electron-lattice phase. However, $\mathrm{W}$ is a strong coupling material and lattice effects may be rapidly generating along the electronic relaxation. Performing simulations at different lattice temperature values we noticed that an ionic temperature effect is indeed observable, increasing slightly the plasmon existence domain. This is attributed to a loosening of the atomic order initially responsible for the material nonplasmonic nature. However the effect is of secondary importance compared to the charge redistribution induced by the electronic temperature within the band structure. We conclude that lattice heating, albeit its influence on atomic order, does not severely impact the calculated electronic structure on the relevant time scales, maintaining a dominant electronic drive for the process.

\section{CONCLUSION}

In conclusion, electron temperature dependent $a b$ initio MD-KG calculations of solid state $\mathrm{W}$ indicate large duty cycle optical tuning upon ultrafast laser irradiation and potential excitation of propagating collective electronic motion for an initially nonplasmonic state. We demonstrate that the evolution of optical properties with $T_{e}$ produces, via the necessary optical conditions, an extension of the predictable existence domain of surface plasmons in the visible range, rendering possible a transient plasmonic phase and a potential plasmonic involvement in LIPSS. This activation occurs on the time scale of the laser pulse where the lattice remains stable. The necessary condition for optical indices was confirmed by time-resolved ellipsometry on excited W surfaces. The initial W state with a structured DOS and a $d$-band pseudogap has a nonplasmonic optical character. The dynamic evolution mechanism following ultrashort laser heating is related to a redistribution of localized $d$ electrons across a chemical potential located in a $d$-band pseudogap, without affected collisional frequencies or population numbers. Since the DOS is not distorted by electronic heating, the changes of plasmon properties, indicated here by corresponding changes of optical conductivities, are principally due to Fermi broadening within a structured $d$ block, extending the transition space for visible frequencies. By depopulating low-lying electronic states in favor of high electronic states around the $d$-block pseudogap, quasiresonant transitions from regions of high DOS are diluted in a continuum of transitions. The dissimilar behaviors of intraand interband absorption events mark thus the evolution of the optical conductivity towards fulfilling resonant conditions. Similar phenomena may occur in other metals exhibiting nonplasmonic characteristics [37], especially $\mathrm{Cr}$ and $\mathrm{Mo}$, as they crystallize in a similar structure. Stronger changes of optical properties [40] are to be expected in the case of transition and noble metals where the electronic structure varies substantially with electronic temperature. The potentiality of a plasmonic state may contribute to the enhancement contrast in the scattering scenarios leading to the formation of LIPSS. However, all these effects have implications for optical tunability and ultrafast switching of plasmonic properties extending beyond the domain of laser nanostructuring and validate concepts of structure engineering for metallic materials. They represent proof-of-principle demonstrations of ultrafast large band optical modulation effects potentially interesting in novel concepts for information transport, plasmon-mediated freespace optical switching, or laser nanostructuring strategies.

\section{ACKNOWLEDGMENTS}

We thank N. Faure and M. Torrent for experimental and computing support. This work was supported by ANR project DYLIPSS (ANR-12-IS04-0002-01) and by LABEX MANUTECH-SISE (ANR-10-LABX-0075) of the Université de Lyon, within the ANR program "Investissements d'Avenir" (ANR-11-IDEX-0007). Calculations used resources from GENCI (project gen7041).
[1] M. P. Desjarlais, J. D. Kress, and L. A. Collins, Phys. Rev. E 66, 025401 (2002).

[2] Z. Chen, B. Holst, S. E. Kirkwood, V. Sametoglu, M. Reid, Y. Y. Tsui, V. Recoules, and A. Ng, Phys. Rev. Lett. 110, 135001 (2013).

[3] H. E. Elsayed-Ali, T. B. Norris, M. A. Pessot, and G. A. Mourou, Phys. Rev. Lett. 58, 1212 (1987).
[4] R. W. Schoenlein, W. Z. Lin, J. G. Fujimoto, and G. L. Eesley, Phys. Rev. Lett. 58, 1680 (1987).

[5] V. Recoules, J. Clérouin, G. Zérah, P. M. Anglade, and S. Mazevet, Phys. Rev. Lett. 96, 055503 (2006).

[6] S. T. Murphy, S. L. Daraszewicz, Y. Giret, M. Watkins, A. L. Shluger, K. Tanimura, and D. M. Duffy, Phys. Rev. B 92, 134110 (2015). 
[7] Z. Lin, L. V. Zhigilei, and V. Celli, Phys. Rev. B 77, 075133 (2008).

[8] Y. V. Petrov, N. Inogamov, and K. Migdal, JETP Lett. 97, 20 (2013).

[9] B. Y. Mueller and B. Rethfeld, Phys. Rev. B 87, 035139 (2013).

[10] N. Rotenberg, A. D. Bristow, M. Pfeiffer, M. Betz, and H. M. van Driel, Phys. Rev. B 75, 155426 (2007).

[11] M. Conforti and G. Della Valle, Phys. Rev. B 85, 245423 (2012).

[12] E. Bévillon, J. P. Colombier, V. Recoules, and R. Stoian, Phys. Rev. B 89, 115117 (2014).

[13] K. F. MacDonald, Z. L. Sámson, M. I. Stockman, and N. I. Zheludev, Nat. Photonics 3, 55 (2009).

[14] B. Luk'yanchuk, N. I. Zheludev, S. A. Maier, N. J. Halas, P. Nordlander, H. Giessen, and C. T. Chong, Nat. Mater. 9, 707 (2010).

[15] A. Hessel and A. A. Oliner, Appl. Opt. 4, 1275 (1965).

[16] M. Birnbaum, J. Appl. Phys. 36, 3688 (1965).

[17] V. Zorba, E. Stratakis, M. Barberoglou, E. Spanakis, P. Tzanetakis, S. H. Anastasiadis, and C. Fotakis, Adv. Mater. 20, 4049 (2008).

[18] B. Dusser, Z. Sagan, H. Soder, N. Faure, J.-P. Colombier, M. Jourlin, and E. Audouard, Opt. Express 18, 2913 (2010).

[19] J. Zhang, M. Gecevičius, M. Beresna, and P. G. Kazansky, Phys. Rev. Lett. 112, 033901 (2014).

[20] B. Öktem, I. Pavlov, S. Ilday, H. Kalaycıŏlu, A. Rybak, S. Yavaş, M. Erdoğan, and F. O. Ilday, Nat. Photonics 7, 897 (2013).

[21] J. E. Sipe, J. F. Young, J. S. Preston, and H. M. van Driel, Phys. Rev. B 27, 1141 (1983).

[22] H. Zhang, J. P. Colombier, C. Li, N. Faure, G. Cheng, and R. Stoian, Phys. Rev. B 92, 174109 (2015).

[23] A. Y. Vorobyev and C. Guo, J. Appl. Phys. 104, 063523 (2008).

[24] J.-P. Colombier, P. Combis, E. Audouard, and R. Stoian, New J. Phys. 14, 013039 (2012)
[25] X. Gonze, B. Amadon, P.-M. Anglade, J.-M. Beuken, F. Bottin, P. Boulanger, F. Bruneval, D. Caliste, R. Caracas, M. Cote, et al., Comput. Phys. Commun. 180, 2582 (2009).

[26] P. Hohenberg and W. Kohn, Phys. Rev. 136, B864 (1964).

[27] W. Kohn and L. J. Sham, Phys. Rev. 140, A1133 (1965).

[28] N. D. Mermin, Phys. Rev. 137, A1441 (1965).

[29] J. P. Perdew, K. Burke, and M. Ernzerhof, Phys. Rev. Lett. 77, 3865 (1996).

[30] M. Torrent, F. Jollet, F. Bottin, G. Zérah, and X. Gonze, Comput. Mater. Sci. 42, 337 (2008).

[31] S. Mazevet, M. Torrent, V. Recoules, and F. Jollet, High Energy Density Physics 6, 84 (2010).

[32] E. Bevillon, J.-P. Colombier, B. Dutta, and R. Stoian, J. Phys. Chem. C 119, 11438 (2015).

[33] J. H. Weaver, C. G. Olson, and D. W. Lynch, Phys. Rev. B 12, 1293 (1975).

[34] P. Romaniello, P. L. de Boeij, F. Carbone, and D. van der Marel, Phys. Rev. B 73, 075115 (2006).

[35] P. E. Hopkins, J. C. Duda, R. N. Salaway, J. L. Smoyer, and P. M. Norris, Nanoscale Microscale Thermophys. Eng. 12, 320 (2008).

[36] C. A. D. Roeser, A. M.-T. Kim, J. P. Callan, L. Huang, E. N. Glezer, Y. Siegal, and E. Mazur, Rev. Sci. Instrum. 74, 3413 (2003).

[37] A. D. Rakić, A. B. Djurišić, J. M. Elazar, and M. L. Majewski, Appl. Opt. 37, 5271 (1998).

[38] J. M. Pitarke, V. M. Silkin, E. V. Chulkov, and P. M. Echenique, Rep. Prog. Phys. 70, 1 (2007).

[39] J. P. Colombier, P. Combis, E. Audouard, and R. Stoian, Phys. Rev. E 77, 036409 (2008).

[40] F. Garrelie, J.-P. Colombier, F. Pigeon, S. Tonchev, N. Faure, M. Bounhalli, S. Reynaud, and O. Parriaux, Opt. Express 19, 9035 (2011). 